\title{
Design and Evaluation of M-Commerce Applications
}

\author{
J.J. Wang, Z. Song, P. Lei, R.E. Sheriff \\ Mobile and Satellite Communications Research Centre, School of Engineering, Design and Technology \\ University of Bradford, Bradford, West Yorkshire, BD7 1DP UK \\ \{jwang2, zsong, p.lei, r.e.sheriff\}@brad.ac.uk
}

\begin{abstract}
Recent advances in wireless technology have increased the number of mobile device users and given pace to the rapid development and deployment of e-commerce to the mobile user. This new type of e-commerce, conducting transactions via mobile terminals, is called mobile commerce (mcommerce). Due to its inherent characteristics such as ubiquity, personalization, flexibility, and dissemination, mobile commerce promises business unprecedented market potential, greater productivity and higher profitability. With this in mind, it is perhaps not surprising that mobile commerce is growing much faster than its fixed counterpart.

Unlike e-commerce, m-commerce is more personalized and there is a need for a novel approach to evaluating m-commerce applications. This paper examines the issues in designing $\mathrm{m}$ commerce applications not only from a technical viewpoint but also from the end users' perspective. Firstly, the enabling mcommerce technologies are presented, viz.: Wireless Application Protocol (WAP) and i-mode mobile Internet service. Two mcommerce application scenarios are then illustrated by making use of the simulation software, Nokia toolkit version 4.0. Then results from the authors' on-line market research into user preferences are presented. Finally, conclusions are drawn on the future directions in wireless and mobile Internet service provision.
\end{abstract}

Keywords: mobile commerce, Wireless Application Protocol (WAP), i-mode, market research

\section{INTRODUCTION}

Driven by a widespread understanding of the Internet's capabilities, the power of e-commerce, and advances in wireless technologies and devices, mobile commerce is rapidly approaching the business forefront. According to independent research findings, m-commerce, which is defined as the commercial transactions conducted through a mobile device over a wireless telecommunication network in a wireless environment $[1,2]$, will soon be a dominant force in business and society. The ITU recently announced that subscriber numbers had passed the 1.6 billion mark worldwide by early 2005, and with half of the mobile devices Internet-enabled. Furthermore, the major research findings by IDC estimate that $80 \%$ of the UK's population will own and regularly use an Internet-enabled phone by 2005 . Worldwide, there were 94.9 million users of m-commerce in 2003 and this is expected to grow to 1.67 billion in 2008, resulting in estimated global revenue of US \$554.37 billion [3]. This phenomenal growth in mobile device ownership along with the integration and interoperability of Wireless Local Access Network (WLAN) and the third generation (3G) technologies is driving: application developers to invent more value-added-services that attract more Internet-enabled mobile users, and researchers to investigate the contextual influence behind m-commerce.

M-Commerce enables a new mode of information exchange and purchases, and it presents an unexplored domain. To consumers, it represents convenience; merchants associate it with a huge earning potential; service providers view it as a large unexplored market; governments look it as a viable and highly productive connection with their constituents [4]. Mobile commerce is more than a mobile and wireless extension of the Web-based e-commerce. It is an entirely new sales and promotion channel and is the enabler for a whole range of new services such as buying air tickets, paying tax, reserving a table in a restaurant. Most importantly, it follows the user and is available anytime and anywhere.

In this paper, the current technologies that apply to mcommerce are discussed - WAP and i-mode, and their status reviewed. According to the characteristics of $\mathrm{m}$-commerce, the paper proposes an ideal m-commerce architecture, and two scenarios are used to illustrate this architecture by simulation. The key findings from the authors' currently running webbased market survey strongly support the results of the simulation. Finally, the paper concludes with suggestions for future work.

\section{State Of The Art Of Mobile Commerce}

In order to allow access to the Web from mobile devices, the WAP promoted by the WAP Forum is optimised for the wireless environment. It is the most popular Internet-enabling technology being adopted by handset manufactures and service providers in Europe and America [5]. Moreover, in Japan there exists a proprietary wireless data and mobile Internet access service promoted by NTT-DoCoMo called imode, which attracted 43 million customers by February 2005 [6].

\section{A. WAP for m-commerce}

The WAP 1.0, published in 1998, is a broadly used and accepted protocol standard designed to enable different kinds of wireless devices to communicate and access the Internet. This was a major diffusion of innovation [7]. The WAP 1.0 architecture is based on a client-server relationship. The WAP client communicates with the WAP gateway by sending its request and receiving the content from the Internet. This architecture's goals were: to provide a layered, scalable and extensible architecture that would support as many wireless networks as possible; to optimize communication for narrowband bearers, such as Short Message Service (SMS); to optimize the use of local device resources, such as memory and power consumption; to provide secure applications for the 
wireless devices; and to provide a programming model for telephony services and integration.

The existence of the gateway in WAP is one of the fundamental differences between the WWW and the WAP programming model. The functionality of the WAP gateway is to translate the request from HyperText Markup Language (HTML)/ HyperText Transfer Protocol (HTTP)/Internet Protocol (IP) to a WAP format, which is better suited for OverThe-Air (OTA) transmissions, and to send the response to the WAP client. Even though the gateway can act as a proxy server and therefore can be anywhere on the network, it usually belongs to a third-party vendor. Taking into account the mobile commerce transaction, this model raises two major concerns: scalability and security. From the scalability point of view, the gateway represents a single point of failure and a bottleneck due to the end-to-end flow control. From the security point of view, as it provides protocol translations and compression of content, it acts as a "built-in man in the middle" and is vulnerable to hackers' attacks. WAP has defined Wireless Transport Layer Security (WTLS), with the aim of solving this problem and providing privacy, data integrity and authentication between mobile phones and WAP gateways. However, WTLS does not provide end-to-end security between client and merchant servers if the merchant server is not equipped with the whole WAP protocol stack [8]. In such a situation, a company such as a bank or a large financial institution has to own and manage a WAP gateway within a trusted environment when they perform the m-commerce transaction procedures.

Due to the problems that come with the gateway, Version 2.0 of WAP suggests not to use the gateway but use instead Internet's standards: eXtensible HTML (XHTML), Transmission Control Protocol (TCP) /IP, HTTP and Transport Layer Security (TLS). The most important goal for WAP 2.0 is to bring the mobile Internet services and standardization closer to Internet standards [9].

\section{B. $i$-mode for Mobile Commerce}

With i-mode, mobile phone users gain easy access to more than 88,000 Internet sites form anywhere, anytime in Japan. As i-mode is not an open standard, the amount of available information and its breadth of use are limited at the moment to Japan and a few experiments around the world, particularly in Europe [6].

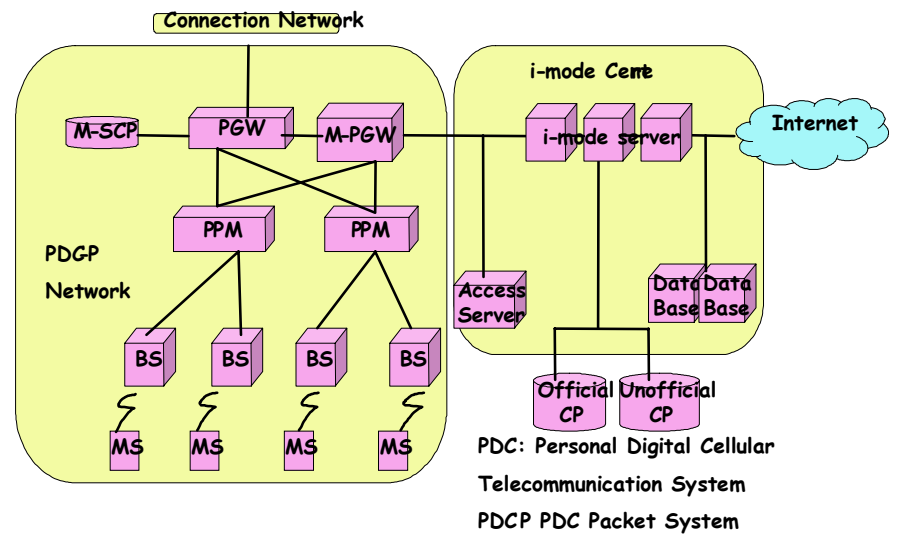

Figure 1. i-mode network architecture i-mode is an enormous client-server network that resides on top of a packet data network. In the case of Japan this is DoCoMo's proprietary Personal Digital Cellular-Packet system (PDC-P), which runs on the PDC telecommunication system, $1500 \mathrm{MHz}$ air interface. However, if and when i-mode is exported to other countries it will need to run on other packet data networks such as General Packet Radio Service (GPRS) and Universal Mobile Telecommunications System (UMTS). Fig. 1 shows the i-mode network architecture.

The Packet Gateway Module (PGW) is responsible for transition to other networks, e.g. to offer a Virtual Private Network (VPN). The Mobile-Service Control Point (M-SCP) deals with the authentication of user data - this point is similar to voice communication. The Packet Processing Module (PPM) between the Base Station (BS) and PGW or M-PGW implements the packet connection with the mobile end-devices. The Mobile Message-Packet Gateway Module (M-PGW) transforms the TCP with the i-mode server and Transport Layer Protocol (TLP) with PPM [10].

The core of this network is the $\mathrm{i}$-mode centre, which serves as the relay for transmissions between the i-mode network and the Internet. When subscribers access i-mode or exchange mail, all of the information passes through the centre. The i-mode server, which represents the contents of "Content Providers", consists of multiple server systems. Each server system is responsible for special tasks. The i-mode system housed in the centre has three main functions: 1) it provides functions to send and receive i-mode mail; 2) it relays information from the servers maintained by both official and unofficial Content Providers (CPs) to "iMenu"; and 3) it relays accesses from imode compatible mobile phones to the Internet [11]. The imode server also has a database that stores the personalized "My Menu" site lists and other information for subscribers. The server sends information associated with subscriber requests every time subscribers access "iMenu" from their mobile phones.

The idea behind i-mode is to use the HTML and HTTP as much as possible. As the i-mode service is not based on WAP, it does not require Wireless Markup Language (WML) to produce front-end applications for mobile phone screens. The i-mode services are based on compact HTML (cHTML) as its markup language and it supports high-resolution colour content, hence one of its successful attributes in attracting subscribers to the wireless Internet compared to WAP access. The main advantage of $i$-mode is the fact that the service is not garden-walled like many of the wireless service portals available in Europe and the USA [5]. Companies and individuals alike are able to create their own compatible Internet sites that can be accessed through the i-mode service.

\section{CurRent Status-Convergence Vs. COMPETITION}

Both WAP and i-mode are popular standards for writing mcommerce applications. The mobile industry is working hard to evolve new applications for m-commerce. Currently, there are numerous companies providing e-commerce services through WAP around the world, and with the huge mobile telephone subscriber base, the potential for m-commerce is tremendous [12]. Even though WAP has had a slow start, both in the device and the applications, it is starting to gather some momentum technically and must now be considered on its 
technical merit. WAP will gradually merge into the HTTP stack, and with more reliable and better networks, the only valid reason for keeping it would be for WML, which caters to small form factor devices. However, WML 2.0 is also expected to merge into XHTML. With mobile networks such as GPRS and UMTS, WAP can access mobile Internet with high data rate, "always-on", and be charged by volume. Most of the effort in redesigning an application for WML is to migrate to an eXtensible ML (XML) model. Once that is done, the actual syntax of the markup language is almost a trivial factor.

At the same time, the growth in the number of i-mode subscribers has far exceeded the expectations held at the time imode was launched. To cope with this and the advance in imode features, DoCoMo has currently updated the i-mode centre with the deployment of the new CiRCUS system, which is an advanced network technology that realizes state-of-the-art reliability. The CiRCUS system can incorporate many servers that are normally idle to solve the glitch occurring in a server and adopt a kind of parent-child structure that utilises master and replica servers by the difference in the level of their authority to create information detours [11].

Of course, i-mode is a serious competitor of WAP 2.0. It has been suggested that WAP may push ahead of i-mode in popularity because WAP has a large community of developers, whereas the tightly NTT-controlled i-mode may be stifled by lack of development mass [13]. As i-mode evolves towards support of XHTML and TCP, with the current WAP evolution, these two technologies will probably converge by evolving imode platforms to support WAP users by enabling WAP phones to access i-mode content. This is being done in Japan, and it is one way for i-mode manufacturers and service providers to sell more equipment and services. By enabling a WAP user to obtain i-mode content, an i-mode service provider could use the product as a way of convincing the WAP user to buy their primary service from the i-mode carrier. Most likely, a gateway function will be used to act as a mediation and conversion access point.

In all probabilities, cHTML will become the common markup language for both i-mode and WAP. This convergence for the technologies will create more opportunities for content providers and the Internet industries, resulting in more applications to m-commerce users, which can then expand the subscriber base in order to grow the revenue stream.

\section{M-COMMERCE APPLICATION SCENARIOS}

The range of applications that characterise m-commerce activities can be largely divided into three categories [14]:

- Content Delivery: The roaming user can be provided with information, alerts, entertainment or advertisements based on their location.

- Financial Transaction: Location-based transactions are perhaps the most complex set of services. The main thrust of the business model is billing based on the customer's location, while a mobile banking application can be customised to quickly deliver secure banking services and provide another value-added benefit.

- Location Based: Driving directions and the tracking of freight, packages and people are a core segment of the emerging Location-Based-Service (LBS) market. Intelligent transportation systems are being introduced around the world, and location-based technology plays a key part in almost every solution.

According to the characteristics of $\mathrm{m}$-commerce business-toconsumer (B2C) commerce, an ideal mobile e-commerce architecture is as shown in Fig. 2. Such a system shall support the following: 1) Client authentication, 2) Server authentication, each party needs to be able to be authenticated by their counterpart to ensure that the counterpart is who they claim to be. 3) Secure channel, i.e., encrypted channel. 4) User-friendly payment scheme supporting micropayment, i.e., buy small things and to pay small amounts of money. The administrative charges for such payments must be small compared to the payments. 5) Receipt delivery, which contains reservation and transaction information. 6) Simple user interface, which is suitable for the mobile users to enter their personal data in any kind of situation $[14,15]$.

In order to test and write applications to be readable by WAP-enabled devices, WAP emulator software can be used on computers. This software can then be used to simulate and test the actions of both WAP gateway and WAP-enabled mobile devices, which are currently the main technology devices utilising WAP. A number of freely available WAP emulation software application packages exist to simulate WAP effects. In the following, two m-commerce execution scenarios are developed using the Nokia WAP emulator version 4.0 and showcased in detail in order to further elaborate Fig. 2 - the ideal $\mathrm{m}$-commerce architecture.

The first scenario, which is illustrated in Fig. 3, is concerned with a LBS service that provides a list of restaurants, which are located near to the current location and match a set of user preferences. This reservation service starts with the request from the browser to its Profile Agent (PA). The PA requests the list of context components such as network type and user location. In the presented example, the user is located in Birmingham; the PA forwards this information to both Network Agent (NA) and Local Restaurant Service Provider (LRSP) and requests the restaurant list from the LRSP. After obtaining the local restaurant report, the PA forwards it to the user together with the user's preference. The PA processes the user's requirement and sends confirmation to the SMS-C. This service is completed with a displayed confirmation on the user's mobile phone from SMS-C. For this reservation service, tourists are a key customer segment requiring the locationbased information, since they are most often found in unfamiliar geographic surroundings.

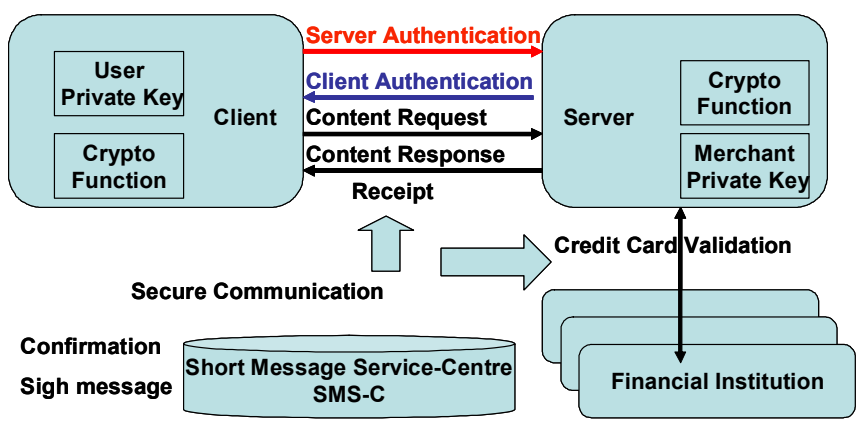

Figure 2. An ideal mobile commerce architecture 


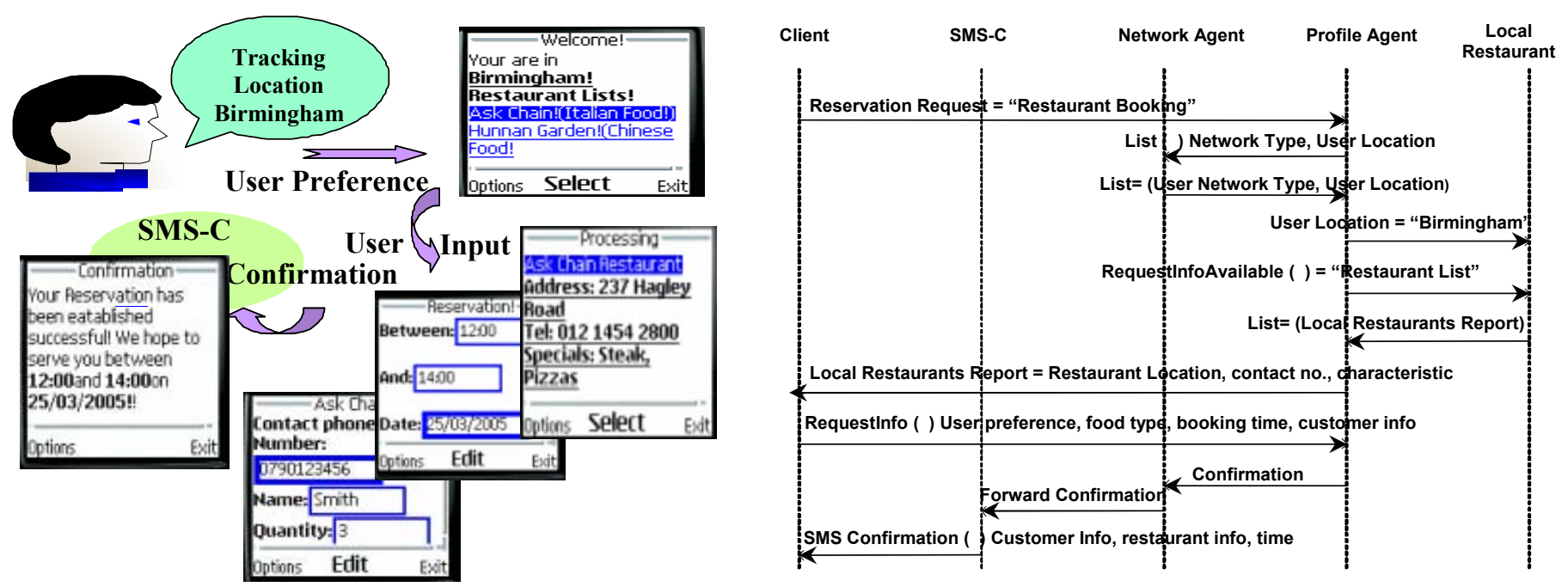

Figure 3. Restaurant reservation procedures and signalling flow
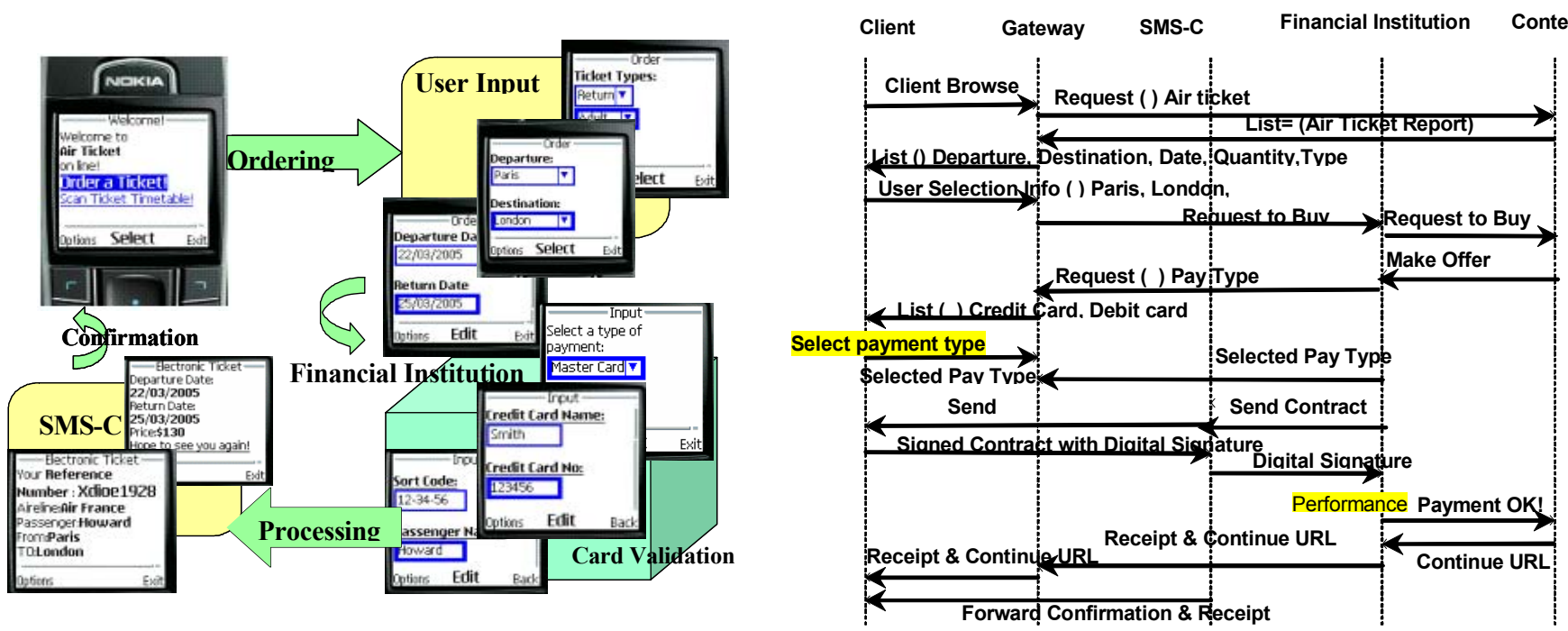

Figure 4. Mobile ticket ordering procedures and signalling flow

The second scenario, illustrated in Fig. 4, presents a whole financial transaction procedure by purchasing an air ticket while mobile. This is more complicated than the first scenario as it involves a money transaction and payment procedure. The initial steps are similar to the first scenario, which inputs a set of user preferences, such as departure time, destination and ticket type. Following this information, the user comes to a secure domain, which hosts financial institutions or banks. This step is a significant part in m-commerce applications. The money transaction will be performed in this secure channel by selecting the payment type. After the user performs the digital signature for the contract sent by the SMS-C which captures all the information about the transaction, the bank will generate a receipt and then sends it together with the Uniform Resource Locator (URL) continuation to the browser via the WAP gateway, and at the same time, the SMS-C sends a mobile ticket with bar-code. This kind of mobile ticket service creates an extra purchase possibility for public transportation tickets via the mobile phone. Even though the scenarios described above are complicated, from the mobile users' point of view, it is transparent and offers the benefit of being able to purchase goods and request services at anytime, anywhere, without the constraints of opening hours and physical distribution points, and most importantly, it is a cashless payment.

From the procedures that have been presented in the two scenarios, it is clear to see that there are two critical procedures that urgently need to be solved: user input usability between the client and server, and credit card payment security, which is performed by financial institutions. As the mobile phone user has to scroll through the information categories available for request and select the category by pressing a key on the phone pad, a wireless device must be easy to use. In this respect, usability could be the critical limitation. Security is still the main concern of business to adapt $\mathrm{m}$-commerce for Intranet and extranet applications. These scenario procedures map onto 
the ideal m-commerce architecture and address the critical components in this system.

\section{WEB-BASED MARKET SURVEY}

The importance of the factors affecting or determining patterns of $\mathrm{m}$-commerce usage has been emphasised in the previous section. Taking this into account, the authors conducted a research study of user preference on mobile services in early 2005 , which is available at [16] in order to investigate the perception and acceptability rates of different mobile services among current and potential users. The study was based primarily in Europe, with more than 200 respondents from different communities, age groups, professions, etc. However, the teenagers/young adult age group (19-30 years) was chosen as being the major focus group, since this generation is known to have the most familiarity with and access to the latest wireless technologies.

The range of different applications offered presents an interesting theme to study. As three categories are addressed above, content delivery has been divided into Information Services and Entertainment Services, which together with Financial Transactions and Location Based Services, provide four types of service to rank by user's preference. One of the most important results of the research was a comparative evaluation of all the above services by the participants. This provides a useful insight into the user's choices and preferences. This is an important input to design content strategy formulation, and could also be an important issue to consider for developing marketing strategy. The research findings show that in spite of all the marketing hype about the range of services that $\mathrm{m}$-commerce promises to provide, $70 \%$ of people still ranked traditional information services as their most important preference. Both entertainment services and financial transactions have been ranked as the least important by one-third of the respondents [17]. Moreover, from the first 200 respondents, 78\% selected "ease of use" and 79\% selected "security" as "important" and "very important" factors, which influenced their adoption of the mobile services. When asked the reasons for interest in buying a $3 \mathrm{G}$ mobile phone, "services available" was the main reason, and followed by the "battery life", "ease of use/functionality" and "handset size/colour appearance".

\section{CONCLUSION AND FUTURE DIRECTIONS}

With the exception of cost, the major factors that influence mobile users are usability and security. The rich content is still the key attraction from the user's perspective. These research findings provide an insight into the user's perception of the telecommunication sector and suggest that interface developers need to consider the application-specific functionality, ease of use and the ability to interact among different user tasks. Application developers should also consider the interaction between the context of the environment, e.g. the location, and the device. A comparative framework for evaluating the usability and security of $\mathrm{m}$-commerce applications is necessary.

There are also many other factors that may influence the adoption of m-commerce, such as lack of killer applications and cultural influences. Having said that, the benefits from mcommerce touch on many aspects of the value chain. For service providers, it can add significant value to their existing voice offerings by adding m-commerce based data services; the integration of a microbrowser into the product line would allow a handset manufacturer to meet the demands of today's advanced service providers; application developers can reach the largest audience when they write their applications in XHTML; ultimately, the subscribers are the most important beneficiaries of $\mathrm{m}$-commerce applications [18].

\section{REFERENCES}

[1] S.J. Barnes, "The mobile commerce value chain: Analysis and Future Developments", International Journal of Information Management, 22(3) , pp.91-110, June 2002.

[2] C. Coursaris and K. Hassanein, "Understanding M-commerce. A consumer-centric Model", Quarterly Journal of Electronic Commerce, 3(3), pp.247-271, 2002.

[3] A. Gunasaekaran and E. Ngai, Decision Support Systems "Special issue on mobile commerce: Strategies, technologies and applications", vol. (35), pp. 187-188.

[4] Wireless Week, "Buying Numbers", pp: 30, 2004.

[5] G. Singh, "Securing the mobile e-conomy", Wireless Week, 2000.

[6] G. Elliott and H. Tang, "The wireless mobile Internet: an international and historical comparison of the European and American Wireless Application Protocol (WAP) and the Japanese iMode ${ }^{\mathrm{TM}}$ service", Internation Journal of Information Technology and Management, 3, Nos. 2/3/4, pp. 268-281, 2004.

[7] NTT DoCoMo Official Website, avaliable at: http://www.nttdocomo.com/corebiz/services/imode/index.html. Accessed on 16, March 2005.

[8] G. Elliott and N, Phillips, Mobile Commerce and Wireless Computing Systems, Pearson Education, Addison-Wesley, 2003.

[9] S. Dixit and T. Wu, Content Networking in the Mobile Internet, $1^{\text {st }}$ ed., John Wiley and Sons, New Jersey, 2004.

[10] I. Cleju, "Security problems in M-commerce using WAP", March 2004 , available at: http://www.cs.joensuu.fi/ icleju/EssaysContributions/WAP/SecurityPro blemsinMcommerceusingWAP.htm Accessed on 16, March 2005.

[11] NTT DoCoMo, "i-mode, an overview," Mobile Communication and Mobile Computing, based partially on the materials of NTT DoCoMo, pp.305-340. available at: http://www.rn.inf.tu-

dresden.de/scripts_lsrn/Lehre/mobile/print_en/18_en.pdf Accessed on 16, March 2005

[12] NTT DoCoMo Report, “CiRCUS-new i-mode operation systemDoCoMo Opens New i-mode Center Equipped with the World's Highest Level Network Technology, "April 2003. avilable at: http://www.nttdocomo.com/files/presscenter/34_No14_Doc.pdf

[13] Auerbach Analysis, "WAP paves the way for m-commerce," Sep, 2001, available http://techupdate.zdnet.com/techupdate/stories/main/0,14179,2813131,0 0.html Accessed on 16, March 2005.

[14] P. Smith, WAP and WML, “An introduction to the mobile Internet, and to creating your own WAP site", Net. Works, UK, 2000.

[15] E.P. Lim and K. Siau, Advances in Mobile Commerce Technologies, $1^{\text {st }}$ ed., Idea Group Inc., UK, 2003.

[16] Available at: http://www.questionpro.com/akira/TakeSurvey?id=202545

[17] R. Das, J.J. Wang and P. Lei, “ A socio-cultural analysis of the present and the future of the m-commerce industry", Handbook of research in mobile business:technical, methodological and social perspective, editor B. Unhelkar, IDEA Group Inc., 2005, in press.

[18] S.S. Nan, Wireless Communications and Mobile Commerce, IRM Press, UK, 2004. 